\title{
Polymer mean spherical approximation for the fluid of flexible hard-sphere Yukawa star molecules
}

\author{
Yu.V.Kalyuzhnyi, M.F.Holovko \\ Institute for Condensed Matter Physics \\ of the National Academy of Sciences of Ukraine, \\ 1 Svientsitskii Str., 79011 Lviv, Ukraine
}

Received February 12, 2002

An extension of the product-reactant Ornstein-Zernike approach (PROZA) for the fluid of flexible star molecules is proposed and the corresponding version of the mean spherical approximation (MSA), the so-called polymer MSA (PMSA), is formulated. Using Baxter-Wertheim factorization technique, an analytical solution of the PMSA for the fluid of star molecules with Yukawa hard-sphere interaction between the molecular segments is derived and closed form analytical expressions for the Helmholtz free energy, chemical potential and equation of state are presented. The structure properties of several different versions of the star fluid model are studied.

Key words: star polymers, polymer mean spherical approximation, association, Yukawa hard-sphere fluid, multidensity Ornstein-Zernike equation

PACS: 05.20.Jj, 05.70.Ce, 64.10. $+h$

\section{Introduction}

Over the past two decades there has been considerable progress in the extension and development of the liquid state integral equation methods for the polymeric fluids. Most of the recently developed theoretical descriptions are based on the extension of the integral equation techniques proposed earlier for the fluid of small interaction-site molecules. These include polymer reference interaction site model (PRISM) theory (see [1] and references therein), polymer Born-Green-Yvon theory $[2,3]$, the theory based on Chandler-Silbey-Ladanyi Percus-Yevick (PY) approach for the site-site fluid $[4,5]$ and the theories grown out from the extensions of the multidensity Ornstein-Zernike (OZ) equation for associating particles [6-11]. We call the latter approach as the product-reactant OZ approach (PROZA). However, the majority of the applications of these theories are restricted to the case of the linear chain molecules. Only recently PRISM theory and the theory based on the 
multidensity version of the PY approximation [12-14] have been utilized for the description of the fluid of star polymers with hard-sphere interaction between the monomeric units.

We gave here an extension of the PROZA developed earlier for the fluid of linear chain molecules [8-11]. The PROZA is a general statistical-mechanical theory for the mixture of reacting species with the product and reactant molecules treated on the same footing. It is represented by the multidensity version of the OZ equation written in terms of the partial correlation functions, which describe correlations between the molecules being in different bonding states. In the previous studies PROZA was formulated for the associating fluid, which in the complete association limit reduces to the fluid of linear chain molecules. In the present study we propose the version of the PROZA, which describe the fluid of associating particles forming star molecules upon association. For the sake of the analytical description the theory is supplemented with the polymer mean spherical approximation (PMSA) closure conditions $[11,15]$ and applied to the fluid of star molecules with Yukawa hard-sphere interaction between the monomeric units. We present an analytical solution of the PMSA for the model at hands and derive closed form analytical expressions for its thermodynamical properties. By way of illustration we consider several different versions of the hard-sphere Yukawa star model.

The paper is organized as follows. In the next section we discuss the details of the model to be studied, present extension of the PROZA and formulate the corresponding version of the PMSA. Section 3 contains expressions for the thermodynamic properties of the system and section 4 gives a solution of the PMSA for Yukawa hard-sphere star fluid model. Numerical results and discussion can be found in section 5 while in section 6 we collect our conclusions.

\section{The model and theory}

We consider the model fluid composed of star-like molecules with a number density $\rho$. Each of the molecule is represented by the collection of freely jointed tangent hard spheres with one central monomer and $n_{a}$ arms, consisting of $m$ hardsphere monomers, which form a flexible chain with one end attached to the central monomer of the molecule. In addition to the hard-sphere interaction all the sites interact, regardless of whether they belong to the same molecule or to the different molecules, via the long range spherically symmetric potential $\Phi_{i j}^{(\mathrm{lr}) a b}(r)$ of arbitrary type. The pair potential $\Phi_{i j}^{a b}(r)$ between the monomers of species $(a, i)$ and $(b, j)$ is of the form

$$
\Phi_{i j}^{a b}(r)=\Phi_{i j}^{(\mathrm{hs}) a b}(r)+\Phi_{i j}^{(\mathrm{lr}) a b}(r),
$$

where $\Phi_{i j}^{(\mathrm{hs}) a b}(r)$ is a hard-sphere potential

$$
\Phi_{i j}^{(\mathrm{hs}) a b}(r)=\left\{\begin{array}{l}
\infty, \quad r<R_{i j}^{a b}=\frac{1}{2}\left(R_{i}^{a}+R_{j}^{b}\right) \\
0, \quad r>R_{i j}^{a b},
\end{array}\right.
$$

and $R_{i}^{a}$ is the hard-sphere diameter. Here the species of the monomers are denoted using two indices $(a, i)$, where $a$ takes the values $c$ or $p$ and denote whether the corre- 
sponding monomer represent the central bead $(c)$ or belong to one of the molecular arms $(p), i$ denote the species of the $p$ - or $c$-type of the monomers. It is assumed, that for the $c$-type of the bead $i$ takes only one value of $i=1$, while for the $p$-type of the bead $i$ takes the values of $1,2, \ldots, m$.

This model can be seen as a limiting case of complete association of the $m+1$ component mixture of hard spheres with $m-1$ components of the $p$-type of the particles having two sticky $A$ and $B$ sites, one component of the $p$-type of the particles with only one sticky $A$ site and one component of the $c$-type of the particles with $n_{a}$ sticky sites $A, B, C, \ldots$. All the sites are randomly distributed on the hardsphere surface and sticky interaction is present only between the site $B$ of the particles of species $(p, i)$ and site $A$ of the particles of species $(p, i+1)$ and between site $A$ of the particles of species $(p, 1)$ and all $n_{a}$ sites $A, B, C, \ldots$ of the particles of species $(c, 1)$. In what follows we will denote the set of all sticky sites of a given particle as $\Gamma$ and subset of sites from the set $\Gamma$ by small Greek letters $\alpha, \beta, \gamma, \ldots$ We assume also the following relation between the densities $\rho_{i}^{a}$ of the above $m+1$-component system

$$
\rho_{i}^{p}=n_{a} \rho_{1}^{c}=n_{a} \rho
$$

The present model of associating fluid with the additional long-range interaction $\Phi_{i j}(r)$ reduces to the original model of the star polymer fluid in the limit of the infinitely strong sticky interaction, i.e. in the limit of $K_{s} \rightarrow \infty$, where $K_{s}$ denotes the strength of the sticky interaction. Thus, the initial macromolecular system can be described using the corresponding version of the liquid state theory for associating fluids.

We start with the multidensity Ornstein-Zernike equation for the associating system at hand, written in the Fourier $k$-space

$$
\hat{\mathbf{h}}_{i j}^{a b}(k)=\hat{\mathbf{c}}_{i j}^{a b}(k)+\sum_{d} \sum_{l} \hat{\mathbf{c}}_{i l}^{a d}(k) \boldsymbol{\rho}_{l}^{d} \hat{\mathbf{h}}_{l j}^{d b}(k),
$$

where the so-called ideal network approximation is utilized. Due to the ideal network approximation, all partial correlation functions, which involve at least one particle with two or more sites bonded, are neglected. Therefore for the matrices $\hat{\mathbf{h}}_{i j}^{a b}, \hat{\mathbf{c}}_{i j}^{a b}$ and $\boldsymbol{\rho}_{i}^{a}$, which appear in the OZ equation (3), we have

$$
\begin{gathered}
\hat{\mathbf{h}}_{i j}^{a b}(k), \hat{\mathbf{c}}_{i j}^{a b}(k)=\left(\begin{array}{ccccc}
\hat{c}_{i_{0} j_{0}}^{a b}(k) & \hat{c}_{i_{0} j_{A}}^{a b}(k) & \ldots & \hat{c}_{i_{0} j_{L}}^{a b}(k) & \ldots \\
\hat{c}_{i_{A} j_{0}}(k) & \hat{c}_{i_{A} j_{A}}^{a b}(k) & \ldots & \hat{c}_{i_{A} j_{L}}^{a b}(k) & \ldots \\
\vdots & \vdots & \ddots & \vdots & \ddots \\
\hat{c}_{i_{K} j_{0}}^{a b}(k) & \hat{c}_{i_{K} j_{A}}^{a b}(k) & \ldots & \hat{c}_{i_{K} j_{L}}^{a b}(k) & \ldots \\
\vdots & \vdots & \ddots & \vdots & \ddots
\end{array}\right), \\
{\left[\boldsymbol{\rho}_{i}^{a}\right]_{00}=\left[\boldsymbol{\rho}_{i}^{a}\right]_{0 L}=\left[\boldsymbol{\rho}_{i}^{a}\right]_{K 0}=\rho_{i}^{a},} \\
{\left[\boldsymbol{\rho}_{i}^{a}\right]_{K L}=\left(1-\delta_{K L}\right) \frac{\sigma_{i_{\Gamma-K-L}}^{a}}{\sigma_{i_{\Gamma-K}}^{a} \sigma_{i_{\Gamma-L}}^{a}}\left(\rho_{i}^{a}\right)^{2}}
\end{gathered}
$$


where the lower indices $K$ and $L$ are taking the values $A, B, C, D, \ldots$ and together with 0 denote the bonded states of the corresponding particles, $\sigma_{i_{\alpha}}^{a}$ is the density parameter, which is related to the density of $\alpha$-bonded particles $\rho_{i_{\alpha}}^{a}$

$$
\sigma_{i_{\alpha}}^{a}=\sum_{\gamma \subset \alpha} \rho_{i_{\gamma}}^{a}
$$

and $\alpha$ denotes the subset of sites from the set $\Gamma$. Here the OZ equation (3) is written in terms of the partial correlation functions, which remain finite in the complete association limit and are related to the original Wertheim's partial correlation functions $h_{i_{\alpha} j_{\beta}}^{(\mathrm{W}) a b}(r)$ and $c_{i_{\alpha} j_{\beta}}^{(\mathrm{W}) a b}(r)$ by

$$
\rho_{i}^{a} h_{i_{\alpha} j_{\beta}}^{a b}(r) \rho_{j}^{b}=\sigma_{i_{\Gamma-\alpha}}^{a} h_{i_{\alpha} j_{\beta}}^{(\mathrm{W}) a b}(r) \sigma_{j_{\Gamma-\beta}}^{b}, \quad \rho_{i}^{a} c_{i_{\alpha} j_{\beta}}^{a b}(r) \rho_{j}^{b}=\sigma_{i_{\Gamma-\alpha}}^{a} c_{i_{\alpha} j_{\beta}}^{(\mathrm{W}) a b}(r) \sigma_{j_{\Gamma-\beta}}^{b} .
$$

Partial correlation functions $\mathbf{c}_{i j}^{a b}(r)$ and $\mathbf{h}_{i j}^{a b}(r)$ can be represented as a sum of the regular and singular terms

$$
\left\{\begin{array}{l}
\mathbf{c}_{i j}^{a b}(r)=\mathbf{C}_{i j}^{a b}(r)+\mathbf{B}_{i j}^{a b} \delta\left(r-R_{i j}^{a b}\right) \\
\mathbf{h}_{i j}^{a b}(r)=\mathbf{H}_{i j}^{a b}(r)+\mathbf{B}_{i j}^{a b} \delta\left(r-R_{i j}^{a b}\right),
\end{array}\right.
$$

where $\mathbf{B}_{i j}^{a b}$ is the matrix with the elements

$$
\begin{aligned}
& B_{i_{\alpha} j_{\beta}}^{p p}=\frac{K_{s} y_{i_{0} j_{0}}^{p p}}{\rho_{i}^{p} \rho_{j}^{p}}\left[\delta_{i, j-1} \delta_{\alpha B} \delta_{\beta A} \sigma_{i_{A}}^{p} \sigma_{j_{B}}^{p}+\delta_{i, j+1} \delta_{\alpha A} \delta_{\beta B} \sigma_{i_{B}}^{p} \sigma_{j_{A}}^{p}\right] \\
& B_{i_{\alpha} j_{\beta}}^{c c}=0, \quad B_{i_{\alpha} j_{\beta}}^{p c}=\frac{K_{s} y_{i_{0} j_{0}}^{p c}}{\rho_{i}^{p} \rho_{j}^{c}} \delta_{i 1} \delta_{\alpha A}\left(1-\delta_{\beta 0}\right) \sigma_{i_{B}}^{p} \sigma_{j_{\Gamma-\beta}}^{c}
\end{aligned}
$$

and $y_{i_{0} j_{0}}^{a b}$ is the contact value of the cavity correlation function $y_{i_{0} j_{0}}^{a b}(r)$ defined by

$$
g_{i_{0} j_{0}}^{a b}(r)=h_{i_{0} j_{0}}^{a b}(r)+1=y_{i_{0} j_{0}}^{a b}(r) \exp \left\{-\beta \Phi_{i j}^{(\mathrm{lr}) a b}(r)\right\} .
$$

The density parameters $\sigma_{i_{\alpha}}^{a}$ follow from the equation (4), where

$$
\rho_{i_{\alpha}}^{a}=\rho_{i_{0}}^{a} \prod_{K \subset \alpha} c_{i_{K}}^{a}
$$

and

$$
\begin{aligned}
c_{i_{A}}^{p} & =\frac{4 \pi\left(R_{i, i-1}^{p p}\right)^{2} \rho_{i}^{p} \rho_{i-1}^{p}}{\sigma_{i_{B}}^{p}} B_{i_{A}(i-1)_{B}}^{p p}, \quad i \neq 1 ; \\
c_{1_{A}}^{p} & =\frac{4 \pi\left(R_{1,1}^{p c}\right)^{2} \rho_{1}^{p} \rho_{1}^{c}}{\sigma_{1_{B}}^{p}} B_{1_{A} 1_{A}}^{p p} ; \\
c_{i_{B}}^{p} & =\frac{4 \pi\left(R_{i, i+1}^{p p}\right)^{2} \rho_{i}^{p} \rho_{i+1}^{p}}{\sigma_{i_{A}}^{p}} B_{i_{B}(i+1)_{A}}^{p p}, \quad i \neq m ; \\
c_{1_{K}}^{p} & =\frac{4 \pi\left(R_{1,1}^{c p}\right)^{2} \rho_{1}^{p} \rho_{1}^{c}}{\sigma_{1_{\Gamma-K}}^{p}} B_{1_{K} 1_{A}}^{c p} .
\end{aligned}
$$


Equation (6) is written under the assumption of the ideal network approximation

$$
c_{i_{\alpha}}^{a}=0 \quad \text { for } \quad \alpha \neq K(\alpha \neq 0) .
$$

Combining (4) and (6) we have

$$
\sigma_{i_{\alpha}}^{a}=\sigma_{i_{0}}^{a} \prod_{K \subset \alpha}\left(1+c_{i_{K}}^{a}\right) .
$$

This relation can be used to simplify the density matrix $\boldsymbol{\rho}_{i}^{a}$. From (8) we get the following equations

$$
\begin{aligned}
\sigma_{i_{\Gamma}}^{a} \sigma_{i_{0}}^{a} & =\sigma_{i_{\Gamma-K}}^{a} \sigma_{i_{K}}^{a}, \\
\sigma_{i_{\Gamma}}^{a}\left(\sigma_{i_{0}}^{a}\right)^{2} & =\sigma_{i_{\Gamma-K-L}}^{a} \sigma_{i_{K}}^{a} \sigma_{i_{L}}^{a},
\end{aligned}
$$

which for the $K L$ elements of the matrix $\boldsymbol{\rho}_{i}^{a}$ give

$$
\left[\boldsymbol{\rho}_{i}^{a}\right]_{K L}=\rho_{i}^{a}\left(1-\delta_{K L}\right) .
$$

The set of the equations (3)-(6) supplemented by the additional relation between the correlation functions $\mathbf{H}_{i j}^{a b}(r)$ and $\mathbf{C}_{i j}^{a b}(r)$ (closure conditions) form a closed set of equations, which can be solved at any degree of association. We are interested in the complete association limit, which can be achieved at $K_{s} \rightarrow \infty$. In this limit we have

$$
\lim _{K_{s} \rightarrow \infty} \rho_{i_{\alpha}}^{a}=\delta_{\alpha \Gamma} \rho_{i}^{a} .
$$

The limiting values of the coefficients $B_{i_{\alpha} j_{\beta}}^{a b}$ follow from the relation (8) written in the form

$$
\sigma_{i_{\Gamma-K}}^{a}=\sigma_{i_{\Gamma}}^{a}-c_{i_{K}}^{a} \sigma_{i_{\Gamma-K}}^{a} .
$$

Substituting into (10) expression for $c_{i_{K}}^{a}$ and taking the limit of $K_{s} \rightarrow \infty$ we have

$$
\begin{aligned}
& B_{i_{\alpha} j_{\beta}}^{p p}=\frac{\delta_{\alpha A} \delta_{\beta B} \delta_{i, j+1}}{4 \pi \rho_{j}^{p}\left(R_{i, i-1}^{p p}\right)^{2}}+\frac{\delta_{\alpha B} \delta_{\beta A} \delta_{i, j-1}}{4 \pi \rho_{j}^{p}\left(R_{i, i+1}^{p p}\right)^{2}}, \\
& B_{i_{\alpha} j_{\beta}}^{c c}=0, \quad B_{i_{\alpha} j_{\beta}}^{p c}=\frac{\delta_{i 1} \delta_{\alpha A} \delta_{\beta K}}{4 \pi \rho_{j}^{c} n_{a}\left(R_{i j}^{p c}\right)^{2}} .
\end{aligned}
$$

Finally to close the set of equations (3) we are utilizing the so-called polymer mean spherical approximation (PMSA)

$$
\begin{cases}\mathbf{H}_{i_{\alpha} j_{\beta}}^{a b}(r)=-\delta_{\alpha 0} \delta_{\beta 0}, & r<R_{i j}^{a b}, \\ \mathbf{C}_{i_{\alpha} j_{\beta}}^{a b}(r)=-\beta \delta_{\alpha 0} \delta_{\beta 0} \Phi_{i j}^{(\operatorname{lr}) a b}(r), & r>R_{i j}^{a b} .\end{cases}
$$

The set of OZ equations (3), together with the PMSA closure conditions (13), relation (5) and expressions for the parameters $B_{i_{\alpha} j_{\beta}}^{a b}$ (11) and (12), represent our polymer mean-spherical ideal network theory for the star polymers with site-site potential $\Phi_{i j}^{a b}(r)(1)$. We note in passing that the extension of the present theory in the case of the multicomponent star fluid is straightforward and reduces to the introduction of the additional indices denoting the molecular species. Since this will make the notation more cumbersome, we restrict the theoretical part of our study to the one-component case. 


\section{Thermodynamics of the polymer mean spherical ideal net- work approximation}

The overall configurational internal energy of the system per unit volume $U$ is

$$
-\beta U=\frac{1}{2} \sum_{a b} \sum_{i j} \rho_{i}^{a} \rho_{j}^{b} \int g_{i j}^{a b}(r)\left[-\beta \Phi_{i j}^{a b}(r)\right] \mathrm{d} \mathbf{r},
$$

where $g_{i j}^{a b}(r)$ is the monomer-monomer radial distribution function, which is related to the partial correlation functions $h_{i_{\alpha} j_{\beta}}^{a b}(r)$ as follows

$$
g_{i j}^{a b}(r)=1+\sum_{\alpha \beta} h_{i_{\alpha} j_{\beta}}^{a b}(r)
$$

Expression (14) can be represented in terms of the total and direct partial correlation functions $h_{i_{\alpha} j_{\beta}}^{a b}(r)$ and $c_{i_{\alpha} j_{\beta}}^{a b}(r)$

$$
\begin{aligned}
-\beta U= & \frac{1}{2} \sum_{a b} \sum_{i j}\left[\boldsymbol{\rho}_{i}^{a} \hat{\mathbf{c}}_{i j}^{a b}(0) \boldsymbol{\rho}_{j}^{b}\right]_{00}+\frac{1}{2} \sum_{a i} \operatorname{Tr}\left\{\boldsymbol{\rho}_{i}^{a}\left[\mathbf{h}_{i i}^{a a}(0)-\mathbf{c}_{i i}^{a a}(0)\right]\right\} \\
& -2 \pi \sum_{a b} \sum_{i j}\left(R_{i j}^{a b}\right)^{2} \operatorname{Tr}\left[\mathbf{B}_{i j}^{a b} \boldsymbol{\rho}_{j}^{b} \mathbf{g}_{j i}^{b a}\left(R_{j i}^{b a}\right) \boldsymbol{\rho}_{i}^{a}\right]
\end{aligned}
$$

where $[\ldots]_{00}$ denotes the 00 element of the matrix inside the brackets, i.e. $[\mathbf{M}]_{00}=$ $\mathbf{M}_{00}$.

We assume, that excess Helmholtz free energy $I=-\beta \Delta A / V=-\beta\left(A-A^{(\mathrm{ref})}\right) / V$ is of the following form

$$
\begin{aligned}
I= & \frac{1}{2} \sum_{a b} \sum_{i j}\left\{\boldsymbol{\rho}_{i}^{a}\left[\hat{\mathbf{c}}_{i j}^{a b}(0)-\hat{\mathbf{c}}_{i j}^{(\mathrm{ref}) a b}(0)\right] \boldsymbol{\rho}_{j}^{b}\right\}_{00} \\
& -\frac{1}{2} \frac{1}{(2 \pi)^{3}} \int\{\operatorname{Tr}[\hat{\mathbf{c}} \boldsymbol{\rho}]+\ln \operatorname{det}[\mathbf{1}-\hat{\mathbf{c}} \boldsymbol{\rho}]\} \mathrm{d} \mathbf{k} \\
& +\frac{1}{2} \frac{1}{(2 \pi)^{3}} \int\left\{\operatorname{Tr}\left[\hat{\mathbf{c}}^{(\mathrm{ref})}(k) \boldsymbol{\rho}\right]+\ln \operatorname{det}\left[\mathbf{1}-\hat{\mathbf{c}}^{(\mathrm{ref})}(k) \boldsymbol{\rho}\right]\right\} \mathrm{d} \mathbf{k},
\end{aligned}
$$

where $\boldsymbol{\rho}, \hat{\mathbf{c}}(k)$ and $\hat{\mathbf{c}}^{(\mathrm{ref})}(k)$ are the matrices with the elements $\delta_{i j} \delta_{a b} \boldsymbol{\rho}_{i}^{a}, \hat{\mathbf{c}}_{i j}^{a b}(k)$ and $\hat{\mathbf{c}}_{i j}^{(\mathrm{ref}) a b}(k), A$ is the total Helmholtz free energy of the system and $A^{(\text {ref })}$ is Helmholtz free energy of the reference system, which is chosen to be a system with $\Phi_{i j}^{(\mathrm{lr}) a b}(r)=0$. Hereafter the subscript "(ref)" denotes the reference system quantities.

Expression (16) for $I$ can be verified using the thermodynamic relation

$$
-\beta U=\beta \frac{\partial I}{\partial \beta} .
$$

The corresponding expressions for the chemical potential and pressure follow from the standard relations

$$
-\beta \rho_{i}^{a} \mu_{i}^{a}=\rho_{i}^{a} \frac{\partial I}{\partial \rho_{i}^{a}}
$$


and

$$
\beta\left(P-P^{(\mathrm{ref})}\right)=I+\beta \sum_{a i} \rho_{i}^{a} \Delta \mu_{i}^{a} .
$$

Using expression (16) for $I$ we have

$$
-\beta \rho_{i}^{a} \Delta \mu_{i}^{a}=\frac{1}{2} \sum_{d l} \rho_{l}^{d}\left\{\boldsymbol{\rho}_{l}^{d}\left[\hat{\mathbf{c}}_{l i}^{d a}(0)-\hat{\mathbf{c}}_{l i}^{(\mathrm{ref}) d a}(0)\right] \boldsymbol{\rho}_{i}^{a}\right\}_{00}-\beta U_{i}^{a},
$$

where $\Delta \mu_{i}^{a}=\mu_{i}^{a}-\mu_{i}^{(\mathrm{ref}) a}$ and

$$
-\beta U_{i}^{a}=\frac{1}{2} \sum_{d l} \rho_{l}^{d} \rho_{i}^{a} \rho_{l}^{d} \int g_{i l}^{a d}(r)\left[-\beta \Phi_{i l}^{a d}(r)\right] \mathrm{d} \mathbf{r} .
$$

Let us consider the virial integral for the long-range part of the total potential, which is defined by

$$
\begin{aligned}
\tilde{J}= & -\frac{1}{6} \beta \sum_{a b} \sum_{i j} \rho_{i}^{a} \rho_{j}^{b} \int_{r>R_{i j}^{a b}} g_{i j}^{a b}(r) \mathbf{r} \nabla \Phi_{i j}^{(\mathrm{lr}) a b}(r) \mathrm{d} \mathbf{r} \\
= & +2 \pi \sum_{a b} \sum_{i j}\left(R_{i j}^{a b}\right)^{2} \operatorname{Tr}\left[\mathbf{B}_{i j}^{a b} \boldsymbol{\rho}_{j}^{b} \mathbf{g}_{j i}^{a b}\left(R_{j i}^{a b}\right) \boldsymbol{\rho}_{i}^{a}\right] \\
& +\frac{2}{3} \pi \sum_{a b} \sum_{i j}\left(R_{i j}^{a b}\right)^{2} \operatorname{Tr}\left[\mathbf{B}_{i j}^{a b} \boldsymbol{\rho}_{j}^{b} \frac{\partial \mathbf{g}_{j i}^{a b}\left(R_{j i}^{b a}\right)}{\partial r} \boldsymbol{\rho}_{i}^{a}\right],
\end{aligned}
$$

where

$$
J=\frac{1}{6} \sum_{a b} \sum_{i j} \operatorname{Tr} \int_{r>R_{i j}^{a b}} \mathbf{r} \nabla \mathbf{c}_{i j}^{a b}(r) \boldsymbol{\rho}_{j}^{b} \mathbf{g}_{j i}^{b a}(r) \boldsymbol{\rho}_{i}^{a} \mathrm{~d} \mathbf{r} .
$$

Following the scheme developed by Høye and Stell [16] we have

$$
J=J_{1}+J_{2}-J_{3},
$$

where

$$
\begin{aligned}
J_{1}= & \frac{1}{6} \sum_{a b} \sum_{i j} \operatorname{Tr} \int \mathbf{r} \nabla \mathbf{c}_{i j}^{a b}(r) \boldsymbol{\rho}_{j}^{b} \mathbf{h}_{j i}^{b a}(r) \boldsymbol{\rho}_{i}^{a} \mathrm{~d} \mathbf{r} \\
= & -\frac{1}{2} \sum_{a i} \operatorname{Tr}\left\{\boldsymbol{\rho}_{i}^{a}\left[\mathbf{h}_{i i}^{a a}(0)-\mathbf{c}_{i i}^{a a}(0)\right]\right\} \\
& -\frac{1}{2} \frac{1}{(2 \pi)^{3}} \int\{\operatorname{Tr}[\hat{\mathbf{c}} \boldsymbol{\rho}]+\ln \operatorname{det}[\mathbf{1}-\hat{\mathbf{c}} \boldsymbol{\rho}]\} \mathrm{d} \mathbf{k}, \\
J_{2}= & \frac{1}{6} \sum_{a b} \sum_{i j} \int\left[\boldsymbol{\rho}_{i}^{a} \mathbf{r} \boldsymbol{\nabla} \mathbf{c}_{i j}^{a b}(r) \boldsymbol{\rho}_{j}^{b}\right]_{00} \mathrm{~d} \mathbf{r}=-\frac{1}{2} \sum_{a b} \sum_{i j}\left[\boldsymbol{\rho}_{i}^{a} \hat{\mathbf{c}}_{i j}^{a b}(0) \boldsymbol{\rho}_{j}^{b}\right]_{00}, \\
J_{3}= & \frac{1}{6} \sum_{a b} \sum_{i j} \operatorname{Tr} \int_{r<R_{i j}^{a b}} \mathbf{r} \nabla \mathbf{c}_{i j}^{a b}(r) \boldsymbol{\rho}_{j}^{b} \mathbf{g}_{j i}^{b a}(r) \boldsymbol{\rho}_{i}^{a} \mathrm{~d} \mathbf{r} \\
= & \frac{1}{3} \pi \sum_{a b} \sum_{i j}\left(R_{i j}^{a b}\right)^{3} \operatorname{Tr}\left[\mathbf{g}_{i j}^{a b}\left(R_{i j}^{a b}\right) \boldsymbol{\rho}_{j}^{b} \mathbf{g}_{j i}^{b a}\left(R_{j i}^{b a}\right) \boldsymbol{\rho}_{i}^{a}\right]
\end{aligned}
$$


Using (15), (24)-(26) and equality $\tilde{J}^{(\text {ref })}=0$ the virial integral $\tilde{J}$ can be written in the following form

$$
\begin{aligned}
\tilde{J}=\tilde{J} & -\tilde{J}^{(\mathrm{ref})}=-\frac{1}{2} \frac{1}{(2 \pi)^{3}} \int\{\operatorname{Tr}[\hat{\mathbf{c}} \boldsymbol{\rho}]+\ln \operatorname{det}[\mathbf{1}-\hat{\mathbf{c}} \boldsymbol{\rho}]\} \mathrm{d} \mathbf{k} \\
& +\frac{1}{2} \frac{1}{(2 \pi)^{3}} \int\left\{\operatorname{Tr}\left[\hat{\mathbf{c}}^{(\mathrm{ref})}(k) \boldsymbol{\rho}\right]+\ln \operatorname{det}\left[\mathbf{1}-\hat{\mathbf{c}}^{(\mathrm{ref})}(k) \boldsymbol{\rho}\right]\right\} \mathrm{d} \mathbf{k}+\beta U \\
& -\frac{1}{3} \pi \sum_{a b} \sum_{i j}\left(R_{i j}^{a b}\right)^{3} \operatorname{Tr}\left[\mathbf{g}_{i j}^{a b}\left(R_{i j}^{a b}\right) \boldsymbol{\rho}_{j}^{b} \mathbf{g}_{j i}^{b a}\left(R_{j i}^{b a}\right) \boldsymbol{\rho}_{i}^{a}-\mathbf{g}_{i j}^{(\mathrm{ref}) a b}\left(R_{i j}^{a b}\right) \boldsymbol{\rho}_{j}^{b} \mathbf{g}_{j i}^{(\mathrm{ref}) b a}\left(R_{j i}^{b a}\right) \boldsymbol{\rho}_{i}^{a}\right] \\
& +\frac{2}{3} \pi \sum_{a b} \sum_{i j}\left(R_{i j}^{a b}\right)^{2} \operatorname{Tr}\left\{\mathbf{B}_{i j}^{a b} \boldsymbol{\rho}_{j}^{b}\left[\frac{\partial \mathbf{g}_{j i}^{b a}\left(R_{j i}^{b a}\right)}{\partial r}-\frac{\partial \mathbf{g}_{j i}^{(\mathrm{ref}) b a}\left(R_{j i}^{b a}\right)}{\partial r}\right] \boldsymbol{\rho}_{i}^{a}\right\} .
\end{aligned}
$$

Comparing expressions (27) and (16) one finds

$$
\begin{aligned}
& I= \tilde{J} \\
&+\frac{1}{2} \sum_{a b} \sum_{i j}\left\{\boldsymbol{\rho}_{i}^{a}\left[\hat{\mathbf{c}}_{i j}^{a b}(0)-\hat{\mathbf{c}}_{i j}^{(\mathrm{ref}) a b}(0)\right] \boldsymbol{\rho}_{j}^{b}\right\}_{00}-\beta U \\
&+\frac{1}{3} \pi \sum_{a b} \sum_{i j}\left(R_{i j}^{a b}\right)^{3} \operatorname{Tr}\left[\mathbf{g}_{i j}^{a b}\left(R_{i j}^{a b}\right) \boldsymbol{\rho}_{j} \mathbf{g}_{j i}^{b a}\left(R_{j i}^{b a}\right) \boldsymbol{\rho}_{i}^{a}-\mathbf{g}_{i j}^{(\mathrm{ref}) a b}\left(R_{i j}^{a b}\right) \boldsymbol{\rho}_{j}^{b} \mathbf{g}_{j i}^{(\mathrm{ref}) b a}\left(R_{j i}^{b a}\right) \boldsymbol{\rho}_{i}^{a}\right] \\
&-\frac{2}{3} \pi \sum_{a b} \sum_{i j}\left(R_{i j}^{a b}\right)^{2} \operatorname{Tr}\left\{\mathbf{B}_{i j}^{a b} \boldsymbol{\rho}_{j}^{b}\left[\frac{\partial \mathbf{g}_{j i}^{b a}\left(R_{j i}^{b a}\right)}{\partial r}-\frac{\partial \mathbf{g}_{j i}^{(\mathrm{ref}) b a}\left(R_{j i}^{b a}\right)}{\partial r}\right] \boldsymbol{\rho}_{i}^{a}\right\}
\end{aligned}
$$

Combining this result and relation (19) one obtains finally

$$
\begin{aligned}
& \beta\left(P-P^{(\mathrm{ref})}\right)= \\
& =\tilde{J}+\frac{1}{3} \pi \sum_{a b} \sum_{i j}\left(R_{i j}^{a b}\right)^{3} \operatorname{Tr}\left[\mathbf{g}_{i j}^{a b}\left(R_{i j}^{a b}\right) \boldsymbol{\rho}_{j}^{b} \mathbf{g}_{j i}^{b a}\left(R_{j i}^{b a}\right) \boldsymbol{\rho}_{i}^{a}-\mathbf{g}_{i j}^{(\mathrm{ref}) a b}\left(R_{i j}^{a b}\right) \boldsymbol{\rho}_{j}^{b} \mathbf{g}_{j i}^{(\mathrm{ref}) b a}\left(R_{j i}^{b a}\right) \boldsymbol{\rho}_{i}^{a}\right] \\
& \quad-\frac{2}{3} \pi \sum_{a b} \sum_{i j}\left(R_{i j}^{a b}\right)^{2} \operatorname{Tr}\left\{\mathbf{B}_{i j}^{a b} \boldsymbol{\rho}_{j}^{b}\left[\frac{\partial \mathbf{g}_{j i}^{b a}\left(R_{j i}^{b a}\right)}{\partial r}-\frac{\partial \mathbf{g}_{j i}^{(\mathrm{ref}) b a}\left(R_{j i}^{b a}\right)}{\partial r}\right] \boldsymbol{\rho}_{i}^{a}\right\} .
\end{aligned}
$$

In the case of the models for which an analytical solution of the PMSA is available, expressions (28) and (29) for the Helmholtz free energy and pressure are much easier to use than the corresponding expressions (16) and (19).

\section{General solution of the multi-Yukawa PMSA for the fluid of star molecules}

Let us consider an analytically solvable case with the long-range part of the potential represented by the sum of the Yukawa terms

$$
\beta \Phi_{i j}^{(\mathrm{lr}) a b}(r)=-\frac{1}{r} \sum_{n} K_{i j}^{(n) a b} \mathrm{e}^{-z_{n} r} .
$$


Recently, general solution of the PMSA for the one-component multi-Yukawa chain fluid was derived [17]. Here we propose an extension of this previously obtained solution for the fluid of the multy-Yukawa star molecules. The general scheme of the present solution is similar to that obtained earlier $[17,18]$. Therefore, we shall omit any details and present here only the final expressions, appropriately modified to be suitable for the system at hand.

The proposed solution is based upon the version of the Baxter-Wertheim factorization technique $[19,20]$ developed by Blum and co-workers [21,22]. We start presenting the set of the OZ equations (3) in a form suitable for factorization

$$
[\mathbf{1}+\hat{\mathbf{h}}(k) \boldsymbol{\rho}]\left[\boldsymbol{\rho}^{-1}-\hat{\mathbf{c}}(k)\right]=\boldsymbol{\rho}^{-1},
$$

where $\hat{\mathbf{h}}(k)$ and $\hat{\mathbf{c}}(k)$ are the matrices with the elements $\hat{\mathbf{h}}_{i j}^{a b}(k)$ and $\hat{\mathbf{c}}_{i j}^{a b}(k)$. Introducing the so-called Baxter factorization $Q$-function $\mathbf{Q}_{i j}^{a b}(r)$ it is possible to separate the initial set of the OZ equations (31) into two sets of equations in which the functions $\mathbf{h}_{i j}^{a b}(r)$ and $\mathbf{c}_{i j}^{a b}(r)$ are decoupled

$$
\left\{\begin{aligned}
\boldsymbol{\rho}^{-1}-\hat{\mathbf{c}}(k) & =\hat{\mathbf{Q}}(k) \boldsymbol{\rho} \hat{\mathbf{Q}}^{T}(-k) \\
\mathbf{1}+\hat{\mathbf{h}}(k) \boldsymbol{\rho} & =\left[\hat{\mathbf{Q}}(k) \boldsymbol{\rho} \hat{\mathbf{Q}}^{T}(-k) \boldsymbol{\rho}\right]^{-1}
\end{aligned}\right.
$$

where $\hat{\mathbf{Q}}(k)$ is the matrix with the elements $\hat{\mathbf{Q}}_{i j}^{a b}(k)$. The corresponding set of equations in the real $r$-space takes the following form

$$
\begin{aligned}
& \mathbf{S}_{i j}^{a b}(|r|)=\mathbf{Q}_{i j}^{a b}(r)-\sum_{d l} \rho_{l}^{d} \int \mathrm{d} r^{\prime} \mathbf{Q}_{i l}^{a d}\left(r^{\prime}\right) \boldsymbol{\alpha}_{d}\left[\mathbf{Q}_{j l}^{b d}\left(r^{\prime}-r\right)\right]^{T}, \\
& \mathbf{J}_{i j}^{a b}(|r|)=\mathbf{Q}_{i j}^{a b}(r)+\sum_{d l} \rho_{l}^{d} \int \mathrm{d} r^{\prime} \mathbf{J}_{i l}^{a d}\left(\left|r^{\prime}-r\right|\right) \boldsymbol{\alpha}_{d} \mathbf{Q}_{l j}^{d b}\left(r^{\prime}\right),
\end{aligned}
$$

where

$$
\mathbf{S}_{i j}^{a b}(r)=2 \pi \int_{r}^{\infty} \mathrm{d} r^{\prime} r^{\prime} \mathbf{c}_{i j}^{a b}\left(r^{\prime}\right), \quad \mathbf{J}_{i j}^{a b}(r)=2 \pi \int_{r}^{\infty} \mathrm{d} r^{\prime} r^{\prime} \mathbf{h}_{i j}^{a b}\left(r^{\prime}\right)
$$

and the factor function $\mathbf{Q}_{i j}^{a b}(r)$ is connected to its Fourier transform $\hat{\mathbf{Q}}_{i j}^{a b}(k)$ via the following relation

$$
\mathbf{Q}_{i j}^{a b}(r)=\frac{1}{2 \pi} \int_{-\infty}^{\infty} \mathrm{d} k\left\{\left[\boldsymbol{\rho}^{-1}\right]_{i j}^{a b}-\hat{\mathbf{Q}}_{i j}^{a b}(k)\right\} \mathrm{e}^{-\mathrm{i} k r} .
$$

In equations (33) and (34), $\boldsymbol{\alpha}_{a}$ is the matrix with the elements $\left[\boldsymbol{\alpha}_{a}\right]_{\alpha \beta}=1-\delta_{\alpha \beta}+$ $\delta_{\alpha 0} \delta_{\beta 0}$ and dimensionality $3 \times 3$ for $a=p$ and $\left(n_{a}+1\right) \times\left(n_{a}+1\right)$ for $a=c$.

Substituting into (36) expression for $\hat{\mathbf{Q}}_{i j}^{a b}(k)$, obtained from (32), and using analytical properties of the factor function $\hat{\mathbf{Q}}_{i j}^{a b}(k)$ together with the closure relation (13) we have

$$
\mathbf{Q}_{i j}^{a b}(r)=\left[\mathbf{q}_{i j}^{a b}(r)+\mathbf{t}_{i j}^{a b}\right] \theta\left(R_{i j}^{a b}-r\right)+\tilde{\mathbf{E}}_{a}^{T} \sum_{n} \tilde{\mathbf{D}}_{i j}^{(n) a b} \mathrm{e}^{-z_{n} r}, \quad r>\lambda_{j i}^{b a}=\frac{1}{2}\left(R_{j}^{b}-R_{i}^{a}\right),
$$


where $\tilde{\mathbf{D}}_{i j}^{(n) a b}$ and $\tilde{\mathbf{E}}_{a}$ are the row vectors

$$
\tilde{\mathbf{D}}_{i j}^{(n) a b}=\left(D_{i_{0} j_{0}}^{(n) a b}, D_{i_{0} j_{A}}^{(n) a b}, D_{i_{0} j_{B}}^{(n) a b}, \ldots\right), \quad \tilde{\mathbf{E}}_{p}=(1,0,0), \quad \tilde{\mathbf{E}}_{c}=(1,0,0, \ldots)
$$

and $\tilde{\mathbf{D}}_{i j}^{(n) a b}$ satisfies the following set of algebraic equations

$$
\frac{2 \pi}{z_{n}} \tilde{\mathbf{K}}_{i j}^{(n) a b}=\sum_{d l} \rho_{l}^{d} \tilde{\mathbf{D}}_{i l}^{(n) a d} \boldsymbol{\alpha}_{d}\left[\hat{\mathbf{Q}}_{j l}^{b d}\left(i z_{n}\right)\right]^{T}
$$

with $\tilde{\mathbf{K}}_{i j}^{(n) a b}=\left(K_{i j}^{(n) a b}, 0,0, \ldots\right)$. In $(37)$ the function $\mathbf{q}_{i j}^{a b}(r)$ is defined in the range $\lambda_{j i}^{b a}<r<R_{i j}^{a b}$ by

$\mathbf{q}_{i j}^{a b}(r)=\frac{1}{2} \tilde{\mathbf{E}}_{a}^{T} \tilde{\mathbf{A}}_{j}^{b}\left(r-R_{i j}^{a b}\right)\left(r-\lambda_{j i}^{b a}\right)+\tilde{\mathbf{E}}_{a}^{T} \tilde{\boldsymbol{\beta}}_{j}^{b}\left(r-R_{i j}^{a b}\right)+\sum_{n} \mathbf{C}_{i j}^{(n) a b}\left(\mathrm{e}^{-z_{n} r}-\mathrm{e}^{-z_{n} R_{i j}^{a b}}\right)$.

Here

$$
\begin{aligned}
\tilde{\boldsymbol{\beta}}_{j}^{b} & =\frac{\pi}{\Delta} R_{j}^{b} \tilde{\mathbf{E}}_{b}+\frac{2 \pi}{\Delta} \sum_{n} \tilde{\boldsymbol{\mu}}_{j}^{(n) b} \\
\tilde{\mathbf{A}}_{j}^{b} & =\frac{2 \pi}{\Delta}\left(\tilde{\mathbf{E}}_{b}+\frac{1}{2} \zeta_{2} \tilde{\boldsymbol{\beta}}_{j}^{b}+\sum_{n} \tilde{\mathbf{M}}_{j}^{(n) b}-\tilde{\boldsymbol{\tau}}_{j}^{b}\right), \\
\mathbf{C}_{i j}^{(n) a b} & =\sum_{d} \sum_{l} \boldsymbol{\gamma}_{i l}^{a d}\left(z_{n}\right) \tilde{\mathbf{E}}_{d}^{T} \tilde{\mathbf{D}}_{l j}^{(n) d b}-\tilde{\mathbf{E}}_{a}^{T} \tilde{\mathbf{D}}_{i j}^{(n) a b}
\end{aligned}
$$

and

$$
\begin{aligned}
s \boldsymbol{\gamma}_{i j}^{a b}(s) & =2 \pi \rho_{j}^{b} \mathbf{G}_{i j}^{a b}(s) \boldsymbol{\alpha}_{b} \\
\tilde{\boldsymbol{\mu}}_{j}^{(n) b} & =\sum_{d l} \rho_{l}^{d} \tilde{\mathbf{C}}_{l}^{(\mu) d}\left(z_{n}\right) \boldsymbol{\alpha}_{d} \tilde{\mathbf{E}}_{d}^{T} \tilde{\mathbf{D}}_{l j}^{(n) d b} \mathrm{e}^{-z_{n} R_{l j}^{d b}}, \\
\tilde{\mathbf{M}}_{j}^{(n) b} & =\sum_{d l} \rho_{l}^{d} \tilde{\mathbf{C}}_{l}^{(M) d}\left(z_{n}\right) \boldsymbol{\alpha}_{d} \tilde{\mathbf{E}}_{d}^{T} \tilde{\mathbf{D}}_{l j}^{(n) d b} \mathrm{e}^{-z_{n} R_{l j}^{d b}}, \\
\mathbf{G}_{i j}^{a b}(s) & =\int_{0}^{\infty} \mathrm{d} r^{\prime} r^{\prime} \mathbf{g}_{i j}^{a b}\left(r^{\prime}\right) \mathrm{e}^{-s r^{\prime}} \\
\tilde{\mathbf{C}}_{l}^{(\mu) a}(s) & =\sum_{d} \sum_{k} \tilde{\mathbf{E}}_{a}\left[\boldsymbol{\gamma}_{l k}^{a d}(s)\right]^{T} \mathrm{e}^{s R_{l k}^{a d}} s\left(R_{k}^{d}\right)^{3} \phi_{1}\left(R_{k}^{d} s\right)+\frac{1}{s^{2}}\left(1+\frac{1}{2} s R_{l}^{a}\right) \tilde{\mathbf{E}}_{a}, \\
\tilde{\mathbf{C}}_{l}^{(M) a}(s) & =\sum_{d} \sum_{k} \tilde{\mathbf{E}}_{a}\left[\boldsymbol{\gamma}^{a d} l k(s)\right]^{T} \mathrm{e}^{s \lambda_{l k}^{a d}}\left(R_{k}^{d}\right)^{2} s \varphi_{1}\left(-s R_{k}^{d}\right)-\frac{1+s R_{l}^{a}}{s} \tilde{\mathbf{E}}_{a}, \\
\tilde{\boldsymbol{\tau}}_{j}^{b} & =\sum_{d l} \rho_{l}^{d} R_{l}^{d} \tilde{\mathbf{E}}_{d} \boldsymbol{\alpha}_{d} \mathbf{t}_{l j}^{d b}
\end{aligned}
$$

Also

$$
\begin{aligned}
& \phi_{1}(x)=\frac{1}{x^{3}}\left[1-\frac{1}{2} x-\left(1+\frac{1}{2} x\right) \mathrm{e}^{-x}\right], \quad \varphi_{1}(x)=\frac{1-x-\mathrm{e}^{-x}}{x^{2}} \\
& \zeta_{m}=\sum_{d l} \rho_{l}^{d} R_{l}^{m}, \quad \Delta=1-\pi \zeta_{3} / 6 .
\end{aligned}
$$


We note in passing, that hereafter all the row vectors of the type $\left(A_{0}, A_{A}, A_{B}, \ldots\right)$ are denoted as $\tilde{\mathbf{A}}$.

It can be seen that all the coefficients of the factor function $\mathbf{q}_{i j}^{a b}(r)$ are determined by the set of unknowns $\tilde{\mathbf{D}}_{i j}^{(n) a b}$ and $\boldsymbol{\gamma}_{i j}^{a b}\left(z_{n}\right)$, which follow from the solution of the set of equations (38) and the equations obtained by differentiating relation (34) with respect to $r$ at $\lambda_{j i}^{b a}<r<R_{i j}^{a b}$ and taking the Laplace transformation of both sides of the resulting equation. We have

$$
\sum_{d} \sum_{l} \gamma_{i l}^{a d}\left(z_{n}\right) \hat{\mathbf{Q}}_{l j}^{d b}\left(\mathrm{i} z_{n}\right)=\mathbf{f}_{i j}^{a b}\left(z_{n}\right) / z_{n}
$$

where

$$
\begin{aligned}
& \mathbf{f}_{i j}^{a b}(s)=\tilde{\mathbf{E}}_{a}^{T}\left[\tilde{\mathbf{A}}_{j}^{b}\left(1+\frac{1}{2} s R_{i}^{a}\right)+\tilde{\boldsymbol{\beta}}_{j}^{b} s\right] \frac{1}{s^{2}} \mathrm{e}^{-s R_{i j}^{a b}} \\
& -\sum_{n} \frac{z_{n}}{s+z_{n}} \mathbf{C}_{i j}^{(n) a b} e^{-\left(s+z_{n}\right) R_{i j}^{a b}}+\mathbf{t}_{i j}^{a b} \mathrm{e}^{-s R_{i j}^{a b}}, \\
& \hat{\mathbf{Q}}_{l j}^{d b}(\mathrm{i} s)=\frac{\delta_{l j} \delta_{d b}}{\rho_{j}^{b}} \boldsymbol{\alpha}_{d}^{-1}-\hat{\boldsymbol{\Omega}}_{l j}^{d b}(s),
\end{aligned}
$$

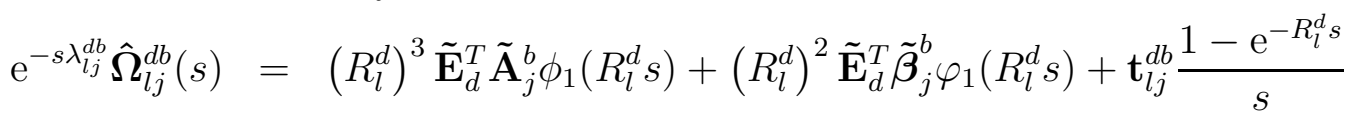

$$
\begin{aligned}
& +\sum_{n}\left\{\mathbf{C}_{l j}^{(n) d b}\left[\frac{\mathrm{e}^{R_{l}^{d} z_{n}}-\mathrm{e}^{-R_{l}^{d} s}}{z_{n}+s}+\frac{\mathrm{e}^{-R_{l}^{d} s}-1}{s}\right] \mathrm{e}^{-z_{n} R_{l j}^{d b}}\right. \\
& \left.+\tilde{\mathbf{E}}_{d}^{T} \tilde{\mathbf{D}}_{l j}^{(n) d b} \frac{\mathrm{e}^{-z_{n} \lambda_{j l}^{b l}}}{z_{n}+s}\right\}
\end{aligned}
$$

The set of equations (38) and (50) have to be solved numerically. This completes our solution of the PMSA for the fluid of star-like Yukawa molecules.

\section{Numerical results}

To illustrate the solution of the PMSA obtained in the previous sections, here we present the numerical results for the structure of three different versions of the start fluid model. We consider a one-component star fluid with the number of arms on each start molecule $n_{a}=4,6$ of the length $m=10$ (model M1), two-component solvent-solute mixture with hard-sphere solvent and solute represented by the star molecules with the number of arms $n_{a}=0,40,100,150$ each of the length $m=3$ (model M2) and infinitely diluted large hard-sphere colloidal particles in the solvent of star molecules with $n_{a}=0,2,4,6,8$ and $m=10$ (model M3). Hard-sphere sizes of the molecular segments were chosen to be the same in the case of the model M1 and M3, while in the case of the model M2 the size of the central bead of the star molecules was chosen to be 10 times larger than that of the arm beads.

In figure 1 we show our results for the radial distribution function (RDF) between the central beads of the two star molecules $g_{c c}(r)$, represented by the model 


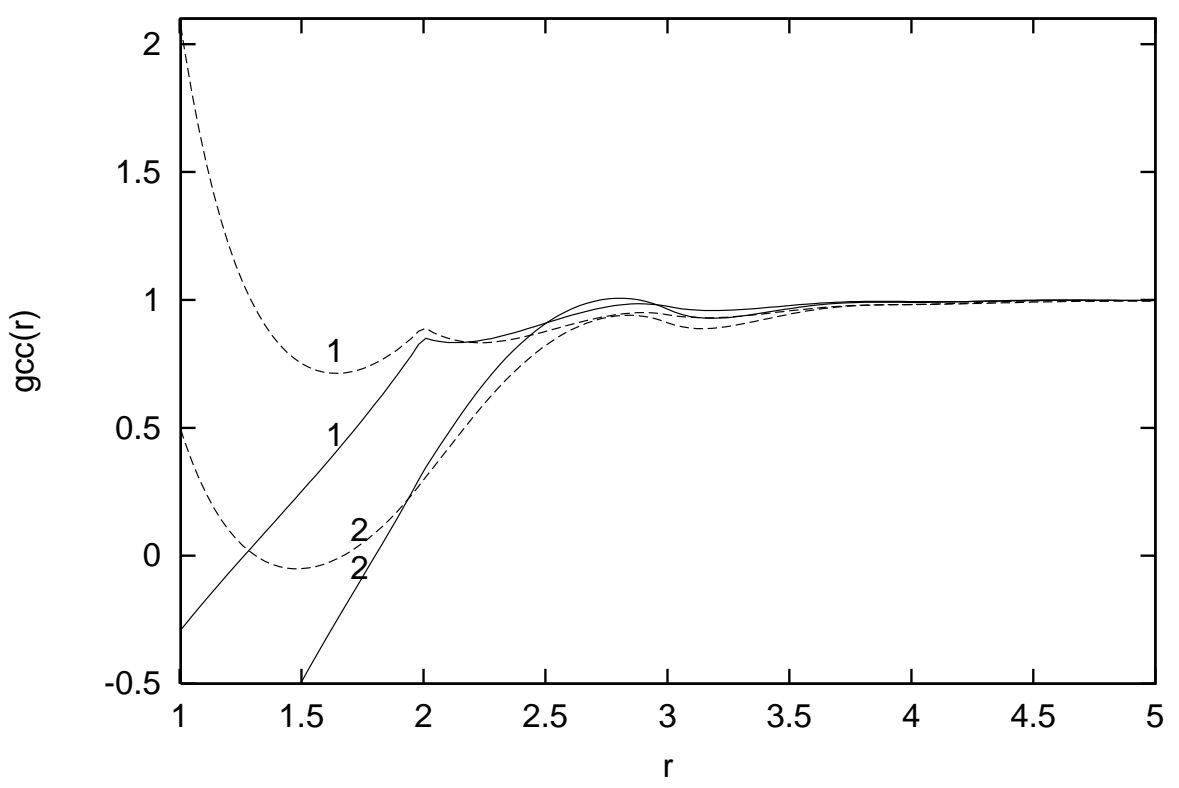

Figure 1. Radial distribution function $g_{c c}(r)$ between the central beads of the two star molecules represented by the model M1 with $m=10$ beads per arm and number of arms $n_{a}=4(1)$ and $n_{a}=6(2)$ at $\eta=0.35, T^{*}=\infty$ (solid lines), $T^{*}=0.4$ (dashed lines).

M1. In addition to the hard-sphere repulsion between all the monomer units of the molecules, we assume here attractive a one-Yukawa interaction with $z R_{c c}=2$, $K_{c c}=\beta$ between the central beads. One can see that $g_{c c}(r)<1$ for $r \leqslant 2.5$, which reflects the screening effects due to the beads of the molecular arms. With the increase of the number of arms this effect becomes more pronounced. Theory predicts negative values of the RDF $g_{c c}(r)$ at the contact. This is an obvious drawback of the theory. With the decrease of the temperature, attractive Yukawa interaction reduces screening effects and the contact values of $g_{c c}(r)$ become positive. This feature points out the possibility of the improvement of the theory using generalized MSA-type of the closure.

In figures 2 and 3 we show the RDF between the central beads of the model M2. In all cases studied it is assumed that total packing fraction $\eta_{T}$ and the packing fraction of the solvent molecules $\eta_{m}$ are constant, i.e. $\eta_{T}=0.35$ and $\eta_{m}=\eta_{T} / 2$. For the version of the model without arms $(m=0)$, RDF $g_{c c}(r)$ has high and narrow first maximum at the contact. This peak appears due to the presence of the hardsphere solvent of small size and reflects the so-called "depletion" effects. With the increase of the number of arms, depletion attraction reduces and due to the screening effects the contact values of the center-center RDF $g_{c c}(r)$ become less than one. For $m=100$ and $m=150, g_{c c}\left(\sigma_{c c}^{+}\right)$takes the negative values. It is interesting to note that for large number of arms $(m=100,150)$ behaviour of the RDF $g_{c c}(r)$ is very similar to the behaviour of the RDF of the spherical particles with soft repulsion of the effective size $R_{\text {eff }}=12.5$ (figure 3 ). 


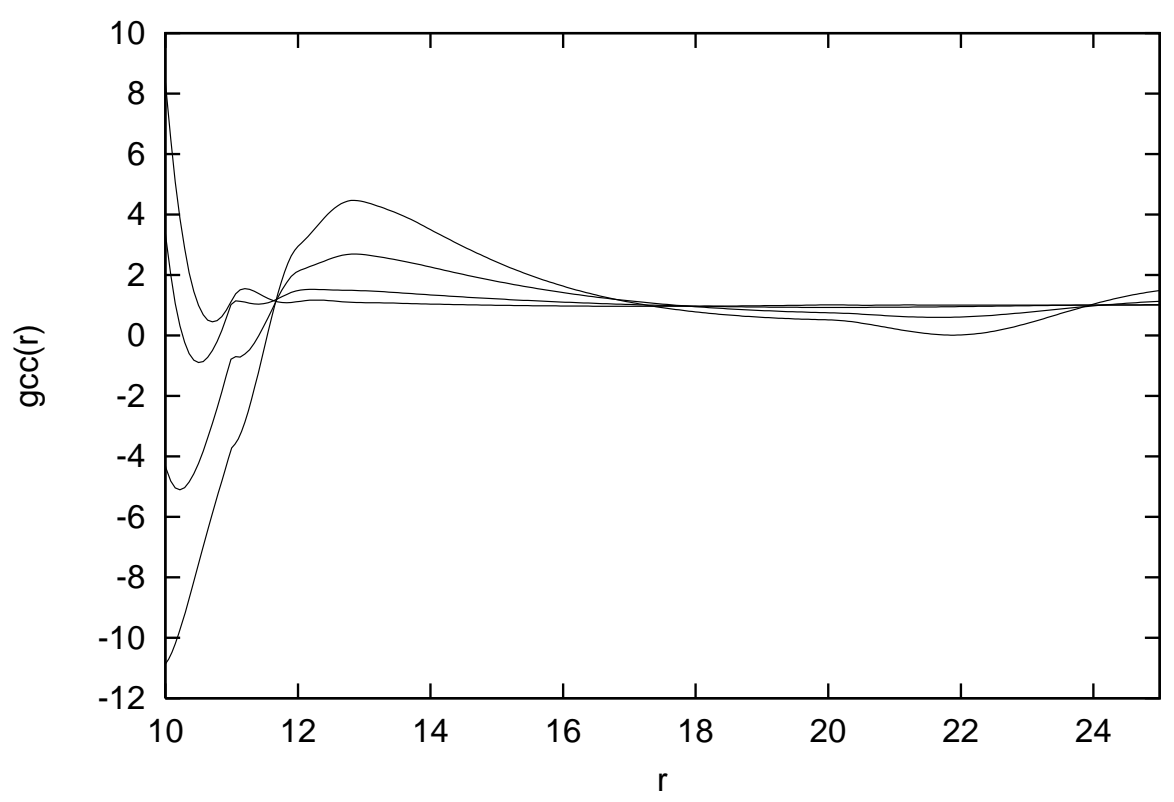

Figure 2. Radial distribution function $g_{c c}(r)$ between the central beads of the two star molecules represented by the model M2 with $m=3$ beads per arm and number of arms $n_{a}=0,40,100,150$ from the top to the bottom at $r=10$. Here $\eta_{\mathrm{T}}=0.35$

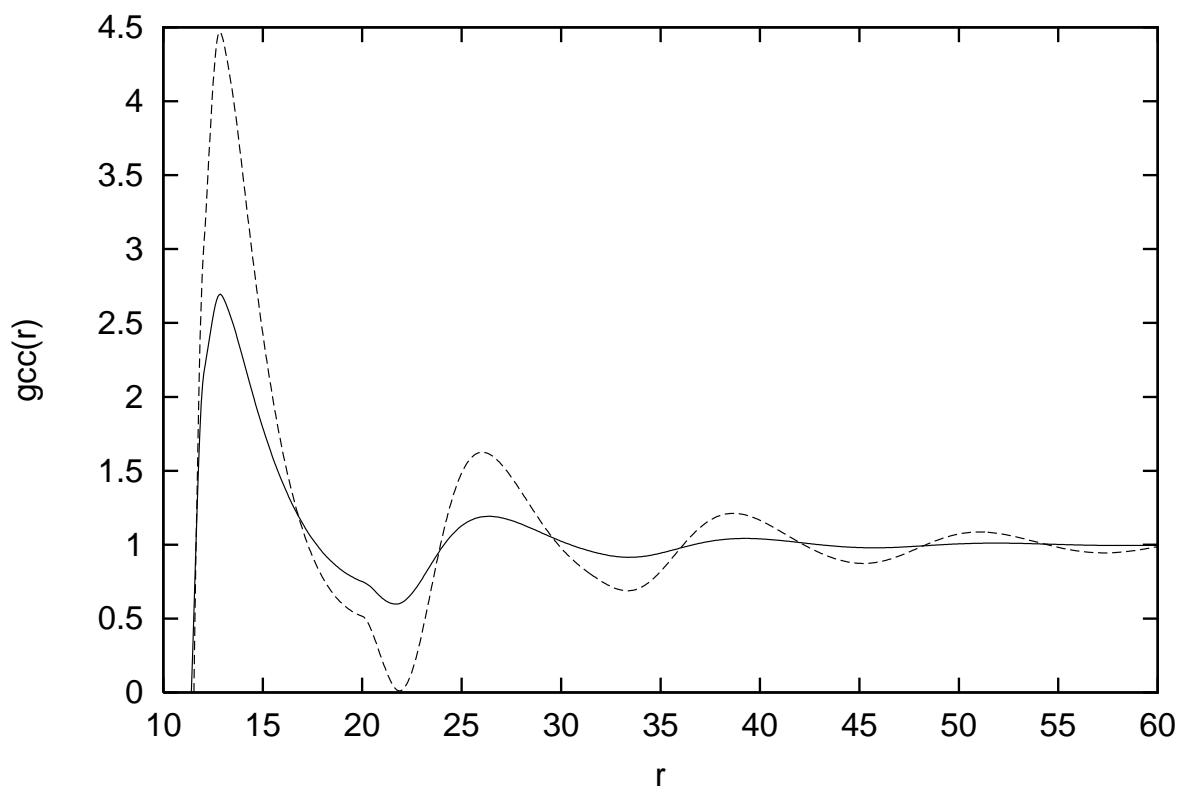

Figure 3. Radial distribution function $g_{c c}(r)$ between the central beads of the two star molecules represented by the model M2 with $m=3$ beads per arm and number of arms $n_{a}=100$ (solid line) and $n_{a}=150$ (dashed line) at $\eta_{\mathrm{T}}=0.35$. 


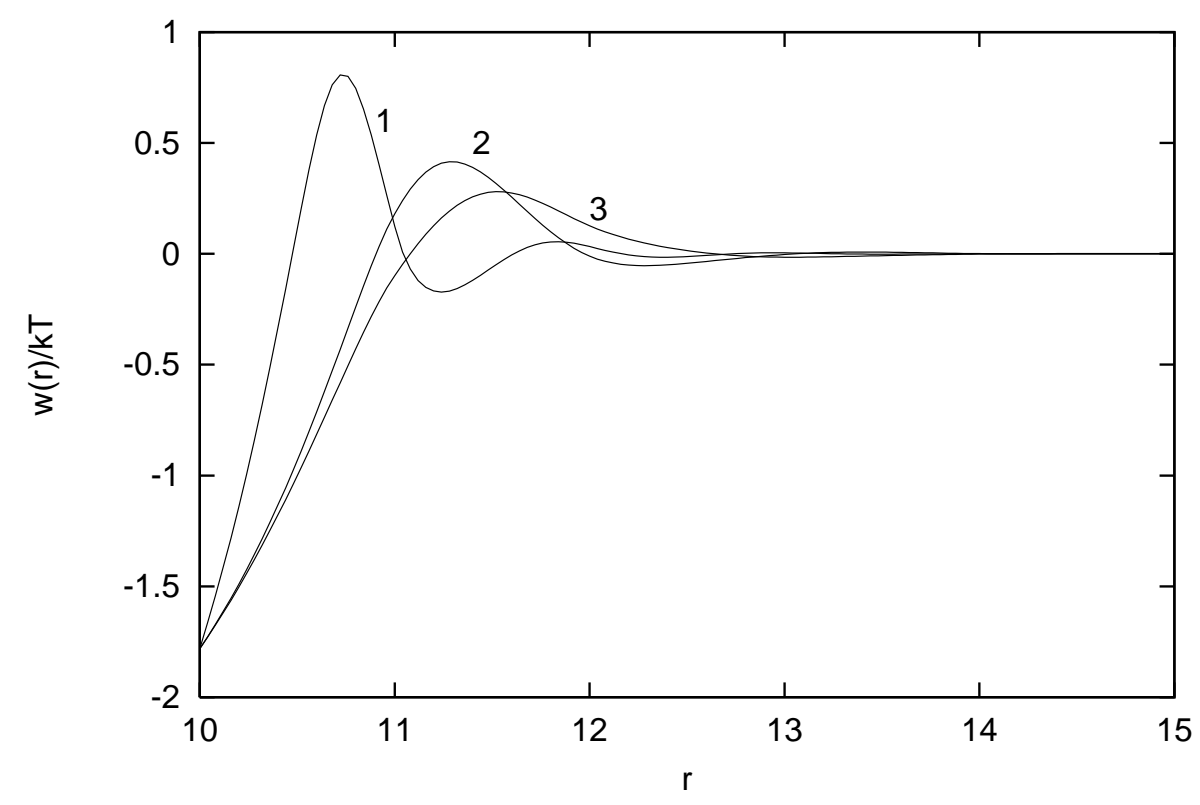

Figure 4. Potential of mean force $\beta \omega(r)$ between two infinitely diluted colloidal particles in a star solvent represented by the model M3 with $m=10$ beads per arm and number of arms $n_{a}=0(1), n_{a}=4(2), n_{a}=12(3)$ at $\eta=0.35$

Finally, in figure 4 we present the mean force potential (MFP) $\beta \omega(r)$ between two infinitely diluted colloidal particles of the model M3. For the version of the model without arms $\left(n_{a}=0\right), \omega(r)$ has deep and narrow attractive well in the vicinity of the contact and potential barrier at a distance $r \approx 10.75$. This shape of the MFP is defined by the presence of depletion effects, which are typical for the colloidal systems. With the increase of the number of arms, the depth of the potential well remains unchanged, while the height of the MFP barrier decreases and its position shifts to the larger distances. This behaviour reflects the changes in the effective sizes of the star molecules, which causes depletion effects.

\section{Conclusions}

In this study we propose an extension of the product-reactant Ornstein-Zernike approach for the fluid of star molecules and formulate the corresponding version of the mean spherical approximation, which we call polymer mean spherical approximation (PMSA). Using Baxter-Wertheim factorization technique we obtain an analytical solution of the PMSA for the fluid of star molecules with Yukawa hardsphere interaction between the molecular segments. Our solution is illustrated by its application to several different versions of the star fluid model. In general, the theory gives qualitatively correct description of the structure of the models at hand. More systematic investigation and comparison with computer simulation results will be carried out in the future studies. 


\section{References}

1. Schweizer K.S., Curro J.G. - In: Advances in Chemical Physics. (Ed. Prigogine I. and Rice S. John Wiley \& Sons, Inc.), 1997 vol. 98, p. 1.

2. Taylor M.P., Lipson J.E.G. // J. Chem. Phys., 1995, vol. 102, p. 2118.

3. Attard P. // J. Chem. Phys., 1995, vol. 102, p. 5411.

4. Kalyuzhnyi Yu.V., Cummings P.T. // J. Chem. Phys., 1995, vol. 105, p. 2011.

5. Kalyuzhnyi Yu.V. // Mol. Phys., 1998, vol. 94, p. 735.

6. Wertheim M.S. // J. Stat. Phys., 1986, vol. 42, p. 459 ; 1986, vol. 42, p. 477.

7. Chang J., Sandler S.I. // J. Chem. Phys., 1995, vol. 102, p. 437.

8. Kalyuzhnyi Yu.V., Lin C.-T., Stell G. // J. Chem. Phys., 1997, vol. 106, p. 1940.

9. Lin C.-T., Kalyuzhnyi Yu.V., Stell G. // J. Chem. Phys., 1998, vol. 108, p. 6513.

10. Kalyuzhnyi Yu.V., Lin C.-T., Stell G. // J. Chem. Phys., 1998, vol. 108, p. 6525.

11. Kalyuzhnyi Yu.V. // Mol. Phys., 1998, vol. 94, p. 735.

12. Grayce C.J., Schweizer K.S. // Macromolecules, 1995, vol. 28, p. 7461.

13. Kalyuzhnyi Yu.V. // Cond. Matter Phys., 1997, vol. 11, p. 71.

14. Duda Y., Garsia I., Trokhymchuk A., Henderson D. // Mol. Phys., 2000, vol. 98, p. 1287.

15. Holovko M.F., Kalyuzhnyi Yu.V. // Mol. Phys., 1991, vol. 73, p. 1145.

16. Høye J.S., Stell G. // J. Chem. Phys., 1977, vol. 67, p. 439.

17. Kalyuzhnyi Yu.V., Lin C.-T., Stell G., Yethiraj A. // J. Mol. Liq., 2001, vol. 92, p. 85.

18. Kalyuzhnyi Yu.V., McCabe C., Cummings P.T. // Fluid Phase Eq., 2002 (in press).

19. Baxter R.J. // J. Chem. Phys., 1968, vol. 49, p. 2770.

20. Wertheim M.S. // J. Math. Phys., 1964, vol. 5, p. 643.

21. Blum L., Høye J. S. // J. Stat. Phys., 1977, vol. 16; 1978, vol. 19, p. 317.

22. Blum L. // J. Stat. Phys., 1980, vol. 22, p. 661. 
Полімерне середньосферичне наближення для рідини гнучких твердосферних юкавівських зіркових молекул

\author{
Ю.В.Калюжний, М.Ф.Головко
}

Інститут фізики конденсованих систем НАН України, 79011 Львів, вул. Свєнціцького, 1

Отримано 12 лютого 2002 p.

Запропоновано узагальнення підходу Орнштейна-Церніке продуктреагент для рідини гнучких зіркових молекул та сформульовано відповідний варіант середньосферичного наближення (CCH), так зване полімерне ССН (ПССН). На основі методу факторизації БакстераВертхайма отримано аналітичний роз'язок ПССН для рідини зіркових молекул з твердосферною юкавівською взаємодією між сегментами молекул та представлені явні аналітичні вирази для вільної енергії, хімічного потенціалу та рівняння стану. Проведено дослідження структурних властивостей кількох різних варіантів моделі рідини зіркових молекул.

Ключові слова: зіркові полімери, полімерне середньосферичне наближення, асоціація, твердосферна юкавівська рідина, рівняння Орнштейна-Церніке

PACS: 05.20.Jj, 05.70.Ce, 64.10.+h 